\title{
Commentary
}

\section{Is lipid oxidation essential for atherogenesis?}

To put it simply, the lipid oxidation hypothesis proposes that high concentrations of low density lipoprotein (LDL) and cholesterol are atherogenic only when they have been oxidised in the artery wall. Most of the evidence supporting this view has accumulated over the past 15 years. ${ }^{1}$ The driving force came from Goldstein and Brown's revelations on the subject of LDL receptors on different cell types, and from the recognition that the lipid laden foam cells of atherosclerosis were not smooth muscle cells, but macrophages. Macrophages are capable of robust oxidative activity as a result of their well developed microbicidal free radical production.

A plausible story can be constructed, mainly on the basis of experiments on cells in culture, that provides a new chemical explanation for the cellular events in atherogenesis. ${ }^{2}$ An abbreviated version is that, first, LDL accumulates preferentially in the arterial wall at sites of chronic haemodynamic endothelial injury. The LDL becomes oxidised in the intima, probably mainly from the oxidative activity of the cells found there. Oxidised LDL then attracts monocytes to emigrate into the wall; it is taken up in large amounts by the macrophages' so-called "scavenger" receptors for abnormal LDL, and a fatty streak, composed of macrophage foam cells, is formed. The powerful oxidative activity of the macrophages causes increased concentrations of oxidised LDL, which eventually become toxic for the macrophages. This causes the development of the lipid core, which is largely a graveyard for dead foam cells. $^{3}$ The mode of death of the macrophages is at least partly by apoptosis. ${ }^{4}$

The advanced lesions always have a collagen rich fibrous cap, laid down by smooth muscle cells, which becomes a barrier to monocyte influx. ${ }^{2}$ At this stage it is only at the periphery or "shoulders" of the plaque that foam cells are found in significant numbers. ${ }^{2}$ There they continue to oxidise LDL and recruit more monocytes. The deeper ones die, thus enlarging the lipid core. ${ }^{3}$ Crucially, this soft peripheral zone is mechanically vulnerable. Rupture at this site gives rise to thrombosis and the worst complications of the disease. ${ }^{5}$

This scenario, based mainly on cell culture experiments, is persuasive only if supported by in vivo evidence. Experiments in animals are largely supportive. Antioxidants, mainly $\alpha$ tocopherol (vitamin E) and probucol, inhibit atherogenesis in several species of animals that are hyperlipidaemic for genetic or dietary reasons. In animals in which lipid cores usually develop (such as Watanabe rabbits), this is inhibited by antioxidants. ${ }^{6}$

What about humans? There is epidemiological evidence suggesting that the incidence of ischaemic heart disease is inversely proportional to vitamin $\mathrm{E}$ intake. One clinical trial, in patients already suffering from ischaemic heart disease, has shown a $75 \%$ decrease in incidence of myocardial infarction in those taking large oral doses of vitamin $\mathrm{E}^{7}$
Perhaps more importantly, human arterial lesions have been shown to contain oxidised LDL and specific oxidised lipids that accurately reflect the findings predicted by the cell culture experiments. ${ }^{8}$

The jigsaw is incomplete, but some already advocate prophylactic vitamin E supplements for susceptible populations. Primary prevention trials in progress will probably settle the question within a few years.

If the lipid oxidation hypothesis proves to be correct, how could it have happened that the disease develops in this way? And why only in industrialised populations? The answer is unlikely to lie simply in inadequate dietary antioxidants. More likely, we are also eating much more oxidisable fat than is natural; and free radical damage from industrial pollutants and smoking may also contribute.

There is as yet no compelling evidence to deny the lipid oxidation hypothesis. In the United Kingdom alone, someone dies every three minutes from the complications of atherosclerosis. The question posed by the title is not just an academic problem, therefore, but a matter of urgent practical importance.

MALCOLM J MITCHINSON

Lecturer, University of Cambridge,

Department of Pathology,

Tennis Court Road,

Cambridge CB2 1QP, United Kingdom

1 Witztum JL, Steinberg D. Role of oxidised low density lipoprotein in atherogenesis. $\mathcal{F}$ Clin Invest 1991;88:1785-92.

2 Mitchinson MJ. The new face of atherosclerosis. $\mathrm{Br} f \mathrm{Clin}$ Pract 1994;48:149-51

3 Ball RY, Stowers EC, Burton JH, Cary NRB, Skepper JN, Mitchinson MJ Evidence that the death of macrophage foam cells contributes to the lipid core of atheroma. Atherosclerosis 1995;114:45-54

4 Mitchinson MJ, Hardwick SJ, Bennett MR. Cell death in atherosclerotic plaques. Curr Opin Lipidol 1996;7:324-9.

5 Felton CV, Crook D, Davies MJ, Oliver MF. Lipid and fatty acid Felton CV, Crook D, Davies MJ, Oliver MF. Lipid and fatty acid
distribution in nonulcerated and ulcerated human aortic plaques. $f$ Vasc distribution in nonulcerated
Med Biol 1993;4:228-34.

6 Braesen JH, Beisiegel U, Niendorf A. Probucol inhibits not only the progression of atherosclerotic disease, but causes a different composition of atherosclerotic lesions in WHHL rabbits. Virchows Archives 1995;426:17988.

7 Stephens NG, Parsons A, Schofield PM, Kelly FJ, Cheeseman KH, Mitchinson MJ, et al. A randomised controlled trial of vitamin $\mathrm{E}$ in patients with coronary disease: The Cambridge Heart Antioxidant Study (CHAOS)
Lancet 1996;347:781-6.

8 Carpenter KLH, Taylor SE, van der Veen C, Williamson BK, Ballantine JA, Mitchinson MJ. Lipids and oxidised lipids in human atherosclerotic lesions at different stages of development. Biochim Biophys Acta 1995;1256:141-

Professor Winder comments:

Dr Mitchinson states that there is " . . no compelling evidence to deny the lipid oxidation hypothesis." I would add, "no compelling evidence to support . ...". The whole thing is a house of cards; very attractive and plausible, the hypothesis may be absolutely valid-but at present the key data are of association and simply not secure. 